\title{
Prevalencia de síndrome de burnout en alumnos del séptimo año de medicina de una universidad privada de Lima, Perú.
}

\author{
Prevalence of burnout syndrome in seventh year medical students from a private university of Lima, \\ Peru.
}

Luis M. Estela-Villa ${ }^{1}$, Carlos R. Jiménez-Román' ${ }^{1}$, Jeremy S. Landeo-Gutiérrez ${ }^{1}$, Jorge D. Tomateo-Torvisco ${ }^{2}$, Johann M. Vega-Dienstmaier².

\begin{abstract}
RESUMEN
El síndrome de burnout (SBO) se ha descrito como una entidad patológica común entre los médicos y que podría originarse durante su formación académica. Objetivo: Determinar la prevalencia y los factores asociados al SBO en alumnos de medicina del séptimo año de la Universidad Peruana Cayetano Heredia (UPCH). Material y Métodos: Se realizó un estudio transversal con los estudiantes de medicina del séptimo año de la UPCH (n=117) durante diciembre del 2010. Se empleó dos encuestas autoadministradas anónimas, una sobre características sociodemográficas y laborales y otra, la escala Maslach Burnout Inventory en su versión Human Services Survey validada en español. Resultados: Se encontró una prevalencia de SBO de 57,2\% (n=59). Más de la mitad de la población presentó niveles medio o alto en despersonalización (DP) $(68,9 \%$ [n=71]) y agotamiento emocional (AE) (67\% [n =69]) y aproximadamente un tercio de los estudiantes tuvieron baja realización personal (RP) (35\% [n=36]). No se encontró asociación estadísticamente significativa entre las variables sociodemográficas y laborales estudiadas y la presencia de SBO. La presencia de enfermedad mental se asoció a niveles altos de DP y AE, mientras la ausencia de actividades extracurriculares se asoció a elevados puntajes en AE. Conclusiones: El SBO estuvo presente en más de la mitad de los internos de medicina de la UPCH. La presencia de enfermedad mental y ausencia de actividades extracurriculares se asociaron a las dimensiones del SBO. (Rev Neuropsiquiatr 2010;73:147-156).
\end{abstract}

PALABRAS CLAVE: Síndrome de burnout, estudiantes de medicina, internado de medicina.

Universidad Peruana Cayetano Heredia. Lima, Perú.

2 Psiquiatra. Facultad de Medicina Alberto Hurtado, Universidad Peruana Cayetano Heredia. Servicio de Neuro-Psiquiatría, Hospital Nacional Cayetano Heredia, Lima, Perú. 


\section{SUMMARY}

Burnout syndrome has been described as a common pathology among physicians and that may begin during medical formation. Objective: To establish the prevalence of burnout and its associated factors in seventh year medical students of Universidad Peruana Cayetano Heredia (UPCH). Materials and Methods: A transversal study was preformed with seventh year medical students from UPCH ( $\mathrm{n}=117)$ during December 2010. Two anonymous self-administered questionnaires were assessed, one of them included sociodemographic and laboral characteristics and the other included the Maslach Burnout Inventory - Human Services Survey version validated in Spanish. Results: The prevalence of burnout was established at $57,2 \%(n=59)$. Over one half of the population had middle or high levels on depersonalization (DP) $(68,9 \%$ [n =71]) and emotional exhaustion (EE) $(67 \%$ [n = 69]) and about one third of students presented low personal accomplishment (PA) (35\% [n=36]). No statistical significance was found between sociodemographic and occupational variables studied and burnout. The presence of mental illness was associated with high levels of DP and EE, while the absence of extracurricular activities was associated with high levels of EE. Conclusions: Burnout is present in more than half of medical interns of UPCH. The presence of mental illness and the absence of extracurricular activities were associated with burnout dimensions.(Rev Neuropsiquiatr 2010;73:147156).

KEYWORDS: Burnout syndrome, medical students, medical internship.

\section{INTRODUCCIÓN}

Durante el curso de la formación médica la salud mental de los estudiantes difiere mucho de sus pares que siguen otras carreras $(1,2)$. En un estudio realizado en nuestro país entre estudiantes del 4to, 5to y 6to año de la facultad de medicina de la Universidad Peruana Cayetano Heredia (UPCH), Galli et al. reportan que un 45,6\% presenta alguna patología psiquiátrica (3), mientras que Saavedra et al. encontraron una prevalencia de $24,6 \%$ de trastornos psiquiátricos en jóvenes limeños de 18 a 24 años (4). La alta presión académica, la sobrecarga laboral, la inestabilidad financiera, la falta de sueño y la cercanía con el paciente, su enfermedad y sus dolencias, tienen un efecto negativo inadvertido en la salud de los estudiantes, encontrándose en este grupo mayor prevalencia de psicopatología, depresión, ansiedad, abuso de sustancias e ideación suicida (5-7).

La presencia del síndrome de burnout (SBO) es una medida de pobre salud mental atribuida al estrés relacionado con el trabajo y es un fenómeno común entre los profesionales de la salud (médicos, enfermeras, etc.) (8-12). Actualmente se define al SBO como una entidad patológica compuesta por tres esferas: agotamiento emocional (AE), despersonalización (DP) y falta de realización personal (RP) (13). El AE se refiere a la sensación de cansancio relacionada con el trabajo; la DP, a una respuesta impersonal, insensible, deshumanizada, poco empática o negativa hacia quienes se atiende o se brinda los servicios; y la RP, a la visión de competencia, logros o éxito en el trabajo. Estas dimensiones pueden coexistir en diferentes grados lo cual hace que el SBO sea una entidad heterogénea y continua (13-15).

Diversos estudios revelan una elevada prevalencia de SBO en médicos generales, especialistas, residentes y recién graduados $(8,10,16-19)$, lo que sugiere que el origen de esta patología ocurre en las facultades de medicina durante el proceso de formación. Un reciente estudio multicéntrico que incluyó 4387 estudiantes de medicina en EE.UU. reporta una prevalencia de SBO de 49,6\% (7). Otros estudios realizados en estudiantes norteamericanos con poblaciones más pequeñas muestran que la prevalencia de SBO fluctúa entre 40$60 \%(20-22)$.

Padecer el SBO durante la formación médica no es un evento benigno. Los estudiantes de medicina con SBO son de 2 a 3 veces más propensos a presentar ideación suicida en comparación a los que no lo presentan (7). Además, estudiantes con puntajes altos en DP, han mostrado asociación con una baja empatía con los pacientes (14). Es decir, el SBO en los estudiantes de medicina se relaciona con problemas de salud mental que llevan al detrimento de su desempeño profesional y a un cuidado sub-óptimo del paciente.

La transición desde la facultad hacia el trabajo es una de las fases más críticas en la profesión médica, es en esta época donde más inciden estresores como la 
sobrecarga laboral, el trabajo en turnos sin horario fijo, el trato con pacientes problemáticos, el contacto directo con la enfermedad, el dolor y la muerte, la falta de especificidad de funciones y tareas -lo que genera conflicto y ambigüedad de rol-, la falta de autonomía y autoridad en el trabajo para poder tomar decisiones y los rápidos cambios tecnológicos (20-23).

En el Perú, el último año de medicina (séptimo año) recibe el nombre de internado médico y se realiza participando de forma activa dentro del sistema público de salud. La labor del interno de medicina consiste en participar en rondas médicas, realizar procedimientos médicos y quirúrgicos, elaborar notas de evolución de cada paciente, participar en turnos de guardia diurna y nocturna, a lo que se añaden numerosos trámites administrativos propios de un sistema de salud como el nuestro.

En la literatura mundial, existen escasos estudios que incluyan como población a estudiantes de medicina del último año que realicen una labor similar a la descrita. En un estudio prospectivo realizado en 110 internos de medicina de la Universidad de Sídney - Australia se observó que al momento de iniciar el internado el 28\% de los internos cumplían con criterios de SBO, en mediciones posteriores durante el año de internado se encontró que dicha cifra aumentaba hasta encontrarse un pico máximo de $75 \%$ (24). Sin embargo, en un estudio transversal realizado en 55 internos de la Universidad del Norte en Colombia se encontró una prevalencia de sólo 9,1\% (25). Por lo tanto, la prevalencia del SBO puede variar ampliamente de un país a otro e incluso dentro del mismo territorio, dependiendo de las características del sistema de salud en que se opera, así como de otros factores organizacionales intrínsecos al hospital que se estudie.

Nuestra hipótesis es que los internos de medicina del país presentan una mayor prevalencia de SBO que la descrita en otras realidades, pues las condiciones laborales actuales a las que están sometidos no son las óptimas, primando el exceso de trabajo y la ausencia de reforzadores, incrementando aún más el riesgo de desadaptación académica y laboral.

El objetivo del estudio fue hallar la prevalencia de SBO en alumnos del séptimo año de la Facultad de Medicina Alberto Hurtado de la Universidad Peruana Cayetano Heredia (UPCH). Además, determinar el nivel de compromiso de las dimensiones componentes del SBO e identificar factores sociodemográficos y laborales que se asocien a la presencia de SBO.

\section{MATERIAL Y MÉTODOS}

Se realizó un estudio descriptivo de corte transversal tomando como población a los estudiantes de medicina que cursaron el séptimo año de estudios durante el período académico 2010, pertenecientes a la Facultad de Medicina Alberto Hurtado de la UPCH ( $n=117)$. Se excluyeron del estudio a los estudiantes que no cumplieron de manera continua todas las rotaciones durante el internado médico 2010, a los que no rindieron el examen nacional de medicina (ENAM) 2010, a los que se negaron a participar en el estudio y a los que entregaron el cuestionario incompleto.

Se obtuvo la autorización del comité de ética de la Universidad Peruana Cayetano Heredia para la realización del estudio. Los participantes fueron ubicados en los diferentes servicios por los que se encontraban rotando durante su última semana de internado 2010. Antes de la inclusión de los participantes se les explicó brevemente el propósito del estudio. Aquellos estudiantes que aceptaron participar firmaron el consentimiento informado respectivo. Posteriormente recibieron dos encuestas, una incluyendo un cuestionario ad-hoc referente a las variables sociodemográficas y laborales que constaba de 20 preguntas; y en la otra el Maslach Burnout Inventory en su versión Human Services Survey (MBIHSS) validada en español.

El MBI-HSS es un instrumento de 22 ítems que es considerado el gold standard para medir el SBO $(8,13-$ 15). Este instrumento tiene subescalas separadas que permiten evaluar cada esfera del SBO: AE, DP y RP. La validez factorial del test en la versión adaptada al español ha sido demostrada (26-29), se reporta un valor alfa de Cronbach de 0,815 para RP, 0,885 para AE y 0,796 para DP (26). No se encontró ningún estudio de validación en nuestro país. El MBI-HSS, ha sido usado en nuestro medio en estudios con médicos generales, especialistas y residentes $(16,17,19)$. Cada ítem del MBIHSS es valorado con una escala tipo Likert, en la que se indica con qué frecuencia han experimentado en el último año la situación descrita por el ítem. La escala de frecuencia recorre siete grados que van de 0 (nunca) a 6 (todos los días). Los scores para cada subescala son definidos como bajo, medio y alto usando puntos de corte establecidos, los cuales se observan en la tabla 1 (13).

Se considera que altos puntajes en las sub-escalas de AE o DP pueden distinguir entre personas que clínicamente padecen SBO y quienes no lo manifiestan 
$(14,15,30)$. En este estudio, se consideró que los estudiantes de medicina presentaban SBO si alcanzaban niveles altos en DP (puntaje mayor o igual a 10) o AE (puntaje mayor o igual a 27) (15).

Los cuestionarios fueron autoadministrados, el tiempo aproximado para completarlos fue de 15 minutos. Para garantizar la confidencialidad de los participantes los cuestionarios se respondieron de manera anónima. Al terminar el llenado de las encuestas, éstas se colocaron en un sobre cerrado hasta el momento de su digitación. Cada formulario tenía un código que correspondía al nombre del participante y fue almacenado en una base de datos digital distinta a la utilizada en el estudio, sólo los investigadores tuvieron acceso a esta información. Los participantes que presentaron SBO fueron identificados con el fin de comunicarles los resultados de su cuestionario y ser orientados sobre el manejo de SBO.

Una vez recolectada la totalidad de encuestas, éstas se digitaron en el paquete estadístico SPSS versión 19.0 para Windows. Se calculó para las variables categóricas la distribución de frecuencia y porcentaje, y para las numéricas la media y desviación estándar (DE). La asociación entre variables categóricas se realizó mediante la prueba de Chi Cuadrado $\left(\mathrm{Chi}^{2}\right)$ y para establecer relación entre variables numéricas y categóricas se utilizó la prueba t de Student. En las pruebas de inferencia se aceptó una significancia menor o igual a 0,05 .

\section{RESULTADOS}

La población total estuvo conformada por 117 estudiantes. Se excluyeron a 2 estudiantes que no iniciaron el año de estudios el $1^{\circ}$ de enero del 2010, 1 estudiante que no cumplió de forma ininterrumpida las rotaciones del internado médico 2010, 8 estudiantes que no desearon participar en el estudio y a los 3 estudiantes responsables del estudio. Finalmente, se contaron con 103 participantes que firmaron el consentimiento informado y llenaron los cuestionarios correctamente.

La media de la edad de los participantes fue de 24,38 ( $D E=1,16)$; en cuanto al sexo, $53,4 \%(n=55)$ fueron varones y 46,6\% $(n=48)$ fueron mujeres. El resto de las características sociodemográficas y laborales se muestran en la tabla 2.

Se encontró una prevalencia de SBO de 57,2\% $(n=59)$ (IC 95\%: 47,7-66,8\%). Al analizar las
Tabla 1. Puntajes por subescala del síndrome de burnout, para categorizar cada subescala y determinar presencia de SBO.

\begin{tabular}{lccc}
\hline Nivel & $\begin{array}{c}\text { Agotamiento } \\
\text { Emocional }\end{array}$ & Despersonalización & $\begin{array}{c}\text { Realización } \\
\text { Personal }\end{array}$ \\
\hline Alto & $27-54$ & $10-30$ & $40-48$ \\
Medio & $19-26$ & $6-9$ & $34-39$ \\
Bajo & $0-18$ & $0-5$ & $33-0$ \\
\hline
\end{tabular}

dimensiones del SBO, se evidenció que más de la mitad de la población presentó niveles medio o alto en DP $(68,9[\mathrm{n}=71])$ y AE $(67 \%$ [n=69]) y aproximadamente un tercio de los estudiantes tuvieron baja RP (35\% [ $n=36])$, como se aprecia en la tabla 3.

En la tabla 4 se muestra el promedio de los resultados obtenidos en las tres subescalas que miden las dimensiones del SBO según las diversas características sociodemográficas y laborales. Al evaluar las tres subescalas del SBO, se observa que en las dimensiones de DP y AE se encuentran más comprometidos aquellos estudiantes que refieren sufrir de una enfermedad mental contra los que no refieren presentar patología alguna ( $p=0,002$ y 0,022, respectivamente). En la dimensión $\mathrm{AE}$, el puntaje promedio es menor en aquellos que realizan actividades extracurriculares frente a los que no las realizan $(\mathrm{p}=0,006)$.

En la tabla 5 observamos la relación entre la presencia o ausencia de SBO y las distintas variables sociodemográficas. No se encontraron diferencias estadísticamente significativas en cuanto a la distribución de las características sociodemográficas y laborales entre los estudiantes que cumplían criterios de SBO y los que no los cumplían. Los internos con SBO obtuvieron notas similares en el ENAM 2010 a los que no presentaron SBO $(15,31$ [DE=1,10] vs. 15,32 [DE $=0,89], t=-0,042, p=0,966)$.

\section{DISCUSIÓN}

Nuestro estudio demostró una alta prevalencia de SBO entre los estudiantes de medicina del séptimo año, donde aproximadamente 6 de cada 10 cumplían criterios de SBO. Este hallazgo es mayor al encontrado en internos de medicina colombianos $(9,1 \%)$ (IC 95\%: $1,44-16,56 \%)$ (25), pero menor al hallado en estudiantes australianos al final de sus rotaciones de internado (75\%) (IC 95\%: 66,9-83,09\%) (24). 
La prevalencia de SBO en nuestros estudiantes es similar a la encontrada en estudios realizados en médicos del país. Jamanca et al. encontraron que un 51,4\% de médicos residentes de un hospital nacional de Lima presentaron SBO (17), mientras que Guido halló que un 56\% de residentes de cirugía y cirujanos generales de tres hospitales de Lima presentaron SBO (16), demostrando que la medicina no sólo desgasta a los médicos, sino también a los estudiantes de medicina debido a su identificación con el ejercicio profesional durante sus prácticas clínicas.
En cuanto a las dimensiones del SBO el nivel alto de DP resultó ser el más prevalente encontrándose en el 46,6\% de los estudiantes. Dicho hallazgo es concordante con el estudio realizado por Willcock et al. quien en su cohorte halló que DP fue la dimensión más prevalente llegando a valores de hasta 63\% (24), sin embargo difiere de lo hallado por Dyrbye et al. donde los más prevalentes fueron niveles altos de AE en estudiantes norteamericanos (7). Este hallazgo puede tener origen en el propio proceso de formación del estudiante. Una de las razones más comunes por las que los estudiantes

Tabla 2. Características de los alumnos del séptimo año de la facultad de medicina Alberto Hurtado Universidad Peruana Cayetano Heredia, 2010.

\begin{tabular}{|c|c|c|c|}
\hline \multicolumn{2}{|c|}{ Variables Cuantitativas } & Media & Desviación estándar \\
\hline \multirow{2}{*}{\multicolumn{2}{|c|}{$\begin{array}{l}\text { Edad } \\
\text { Nota ENAM }\end{array}$}} & 24,38 & 1,16 \\
\hline & & 15,31 & 1,01 \\
\hline \multicolumn{2}{|l|}{ Despersonalización } & 9,41 & 5,82 \\
\hline \multicolumn{2}{|l|}{ Cansancio Emocional } & 24,65 & 10,94 \\
\hline \multicolumn{2}{|l|}{ Realización Personal } & 35,85 & 6,12 \\
\hline \multicolumn{2}{|c|}{ Variables Cualitativas } & $\begin{array}{c}\text { Frecuencia } \\
(N=103)\end{array}$ & $\begin{array}{c}\text { Porcentaje } \\
\text { (\%) }\end{array}$ \\
\hline \multirow{2}{*}{ Sexo } & Masculino & 55 & 53,4 \\
\hline & Femenino & 48 & 46,6 \\
\hline \multirow{2}{*}{ Estado Civil } & Soltero(a) & 102 & 99,0 \\
\hline & Casado(a) & 1 & 1,0 \\
\hline \multirow{2}{*}{ Relación Sentimental } & Sí & 52 & 50,5 \\
\hline & No & 51 & 49,5 \\
\hline \multirow{2}{*}{ Hïjos } & No & 101 & 98,1 \\
\hline & Sí & 2 & 1,9 \\
\hline \multirow{2}{*}{ Convivencia } & Padres/Otros & 97 & 94,2 \\
\hline & Solo & 6 & 5,8 \\
\hline \multirow{2}{*}{$\begin{array}{l}\text { Actividad } \\
\text { Extracurricular }\end{array}$} & No & 81 & 78,6 \\
\hline & Sí & 22 & 21,4 \\
\hline \multirow{2}{*}{ Consumo de Alcohol } & Nunca a 1 vez/mes & 70 & 68,0 \\
\hline & Sí 1 vez/semana a más & 33 & 32,0 \\
\hline \multirow{2}{*}{ Consumo de Tabaco } & No & 71 & 68,9 \\
\hline & Sí & 32 & 31,1 \\
\hline \multirow{2}{*}{ Remuneración } & Sí & 91 & 88,3 \\
\hline & No & 12 & 11,7 \\
\hline \multirow{2}{*}{ Horas de Trabajo } & Hasta 8 horas & 14 & 13,6 \\
\hline & Más de 8 horas & 89 & 86,4 \\
\hline \multirow{2}{*}{ Curso desaprobado } & No & 92 & 89,3 \\
\hline & Sí & 11 & 10,7 \\
\hline \multirow{2}{*}{$\begin{array}{l}\text { Horas de estudio } \\
\text { semanales }\end{array}$} & Menos de 10 horas & 45 & 43,7 \\
\hline & 10 horas o más & 58 & 56,3 \\
\hline \multirow{2}{*}{ Horas de sueño diario } & Menos de 6 horas & 54 & 52,4 \\
\hline & 6 horas o más & 49 & 47,6 \\
\hline \multirow{2}{*}{ Enfermedad Mental } & No & 95 & 92,2 \\
\hline & Sí & 8 & 7,8 \\
\hline \multirow{2}{*}{ Relación con el equipo } & Bueno/Muy bueno & 97 & 94,2 \\
\hline & Regular & 6 & 5,8 \\
\hline
\end{tabular}


Tabla 3. Distribución de resultados de los tres componentes del síndrome de burnout y prevalencia de síndrome de burnout en alumnos del séptimo año de la Facultad de Medicina Alberto Hurtado - Universidad Peruana Cayetano Heredia, 2010.

\begin{tabular}{ccc}
\hline & $\begin{array}{c}\text { Frecuencia } \\
(\mathbf{N}=\mathbf{1 0 3})\end{array}$ & $\begin{array}{c}\text { Porcentaje } \\
(\mathbf{\%})\end{array}$ \\
\hline Agotamiento Emocional & & \\
Alto & 42 & 40,8 \\
Medio & 27 & 26,2 \\
Bajo & 34 & 33,0 \\
Despersonalización & & \\
Alto & 48 & 46,6 \\
Medio & 23 & 22,3 \\
Bajo & 32 & 31,1 \\
Realización Personal & & \\
Alto & 31 & 30,1 \\
Medio & 36 & 35,0 \\
Bajo & 36 & 57,2 \\
Síndrome de Burnout & & 42,8 \\
Sí & 59 & \\
No & 44 & \\
\hline
\end{tabular}

dicen haber escogido la carrera médica es por "tener un interés en ayudar a las personas”, sin embargo, se ha descrito que durante el entrenamiento en las facultades de medicina ocurre un declive en la empatía y humanitarismo de sus estudiantes (31). Estas actitudes negativas, que se desarrollan como consecuencia de las experiencias de los estudiantes y su entorno, inicialmente pueden servir como amortiguadores contra la ansiedad, temor al fracaso y exposición al sufrimiento humano, pero luego pueden llegar a comprometer su profesionalismo $(1,32)$.

Identificar los factores de riesgo para desarrollar SBO supone la oportunidad de realizar intervenciones para prevenir los resultados negativos de esta patología. Dyrbye et al. reportan que 26\% de los estudiantes que estuvieron desgastados al inicio de su estudio fueron recuperados dentro del siguiente año (7), lo que indica que el SBO es reversible. En nuestro estudio, se observó mayor presencia de SBO en los que vivían solos, no realizaban actividad extracurricular, consumían más de una vez a la semana alcohol, no recibían remuneración, trabajaban más de 8 horas diarias, presentaban alguna enfermedad mental y tenían relaciones malas-regulares con el equipo de trabajo. Sin embargo, ninguna de estas asociaciones fue estadísticamente significativa, lo que difiere con lo encontrado por Dyrbye et al., quienes refieren que características personales, eventos negativos en la vida y el ambiente de trabajo/estudio se relacionan independiente con mayor presencia de SBO (23). Se requerirían estudios con mayor número de participantes en nuestra población para confirmar o refutar estas posibilidades.

Al evaluar los promedios de los puntajes obtenidos en cada dimensión hallamos asociación significativa entre presencia de enfermedad mental y niveles altos de DP y AE, mientras la ausencia de actividades extracurriculares se asoció a elevados puntajes en AE. La presencia de enfermedad mental supone un estado vulnerable a cualquier fuente de estrés (33). Conocemos por los estudios de Galli et al. y Rodas et al. que podría existir una mayor proporción de estudiantes de medicina con enfermedad mental en nuestra población $(3,6)$; sin embargo, muchos de ellos pueden no haber buscado ayuda psiquiátrica, ni haber sido diagnosticados debidamente hasta el momento.

La limitación principal de nuestro estudio es haber obtenido los resultados de forma transversal, lo cual excluye una evaluación de temporalidad y causalidad de las relaciones observadas. A pesar de que la tasa de 
Prevalencia de síndrome de burnout en alumnos del séptimo año de medicina de una universidad privada

Tabla 4. Resultados en las escalas de DP, AE y RP según variables estudiadas en alumnos del séptimo año de la Facultad de Medicina Alberto Hurtado - Universidad Peruana Cayetano Heredia, 2010.

\begin{tabular}{|c|c|c|c|c|c|c|c|c|c|}
\hline \multirow[b]{2}{*}{ Variable } & \multicolumn{3}{|c|}{ Valor Despersonalización } & \multicolumn{3}{|c|}{$\begin{array}{c}\text { Valor Cansancio } \\
\text { Emocional }\end{array}$} & \multicolumn{3}{|c|}{$\begin{array}{c}\text { Valor Realización } \\
\text { Personal }\end{array}$} \\
\hline & Media & $\mathrm{DE}$ & $\mathrm{p}^{*}$ & Media & $\mathrm{DE}$ & $\mathrm{p}^{*}$ & Media & $\mathrm{DE}$ & $\mathrm{p}^{*}$ \\
\hline \multicolumn{10}{|l|}{ Sexo } \\
\hline Masculino & 9,95 & 5,78 & \multirow[t]{2}{*}{,318 } & 23,44 & 11,91 & \multirow[t]{2}{*}{,230 } & 35,76 & 6,92 & \multirow[t]{2}{*}{,873 } \\
\hline Femenino & 8,79 & 5,86 & & 26,04 & 9,65 & & 35,96 & 5,13 & \\
\hline \multicolumn{10}{|c|}{ Relación Sentimental } \\
\hline Sí & 9,46 & 6,02 & \multirow[t]{2}{*}{,925 } & 23,08 & 10,68 & \multirow[t]{2}{*}{,141 } & 36,46 & 5,71 & \multirow[t]{2}{*}{,312 } \\
\hline No & 9,35 & 5,66 & & 26,25 & 11,08 & & 35,24 & 6,51 & \\
\hline \multicolumn{10}{|l|}{ Convivencia } \\
\hline Padres/Otros & 9,36 & 5,86 & \multirow[t]{2}{*}{, 744} & 24,55 & 10,84 & \multirow[t]{2}{*}{,700 } & 35,94 & 6,19 & \multirow[t]{2}{*}{, 579} \\
\hline Solo & 10.17 & 5,57 & & 26,33 & 13,63 & & 34,50 & 5,28 & \\
\hline \multicolumn{10}{|c|}{ Actividad Extracurricular } \\
\hline Sí & 8,00 & 4,53 & \multirow[t]{2}{*}{,202 } & 19,00 & 8,93 & \multirow[t]{2}{*}{,006 } & 36,73 & 5,51 & \multirow[t]{2}{*}{,454 } \\
\hline No & 9,79 & 6,09 & & 26,19 & 10,98 & & 35,62 & 6,29 & \\
\hline \multicolumn{10}{|l|}{ Consumo Alcohol } \\
\hline $\begin{array}{l}1 \text { vez al mes o } \\
\text { menos }\end{array}$ & 8,91 & 6,081 & \multirow[t]{2}{*}{,212 } & 23,66 & 10,56 & \multirow[t]{2}{*}{,181 } & 35,69 & 6,535 & ,686 \\
\hline $\begin{array}{l}1 \text { vez a la } \\
\text { semana o más }\end{array}$ & 10,45 & 5,154 & & 26,76 & 11,60 & & 36,21 & 5,219 & \\
\hline Consumo Tabacc & & & & & & & & & \\
\hline Sí & 8,97 & 4,93 & ,610 & 26,03 & 9,80 & 393 & 36,47 & 6,20 & ,497 \\
\hline No & 9,61 & 6,20 & & 24,03 & 11,43 & & 35,58 & 6,12 & \\
\hline Remuneración & & & & & & & & & \\
\hline Sí & 9,24 & 5,70 & ,428 & 24,51 & 11,02 & ,713 & 35,68 & 6,09 & ,432 \\
\hline No & 10,67 & 6,79 & & 25,75 & 10,73 & & 37,17 & 6,46 & \\
\hline Horas de Trabajc & & & & & & & & & \\
\hline Más de 8 & 9,33 & 5,94 & ,721 & 25,15 & 10,81 & ,249 & 35,96 & 5,94 & ,676 \\
\hline Menos de 8 & 9,93 & 5,20 & & 21,50 & 11,67 & & 35,21 & 7,43 & \\
\hline Curso Desaprobc & & & & & & & & & \\
\hline Sí & 11,27 & 5,39 & ,263 & 26,82 & 10,11 & ,490 & 34,82 & 6,82 &, 555 \\
\hline No & 9,18 & 5,86 & & 24,39 & 11,06 & & 35,98 & 6,06 & \\
\hline Horas de Estudio & & & & & & & & & \\
\hline Más de 10 & 8,53 & 5,33 & ,084 & 23,64 & 10,98 & 289 & 36,17 & 6,33 & ,288 \\
\hline Menos de 10 & 10,53 & 6,28 & & 25,96 & 10,88 & & 35,44 & 5,90 & \\
\hline Horas de Sueño & & & & & & & & & \\
\hline Menos de 6 & 9,91 & 6,12 & ,363 & 26,48 & 10,74 & ,075 & 36,02 & 6,41 & ,777 \\
\hline Más de 6 & 8,86 & 5,49 & & 22,63 & 10,92 & & 35,67 & 5,85 & \\
\hline Enfermedad Mer & & & & & & & & & \\
\hline Sí & 15,38 & 7,93 & ,002 & 33,13 & 8,31 & ,022 & 34,38 & 7,69 & ,479 \\
\hline No & 8,91 & 5,366 & & 23,94 & 10,87 & & 35,98 & 6,00 & \\
\hline Relación con Eq & & & & & & & & & \\
\hline Regulares & 9,33 & 4,41 & ,974 & 30,83 & 7,22 & ,155 & 35,17 & 5,98 & ,778 \\
\hline Buenas/Muy & 9,41 & 5,91 & & 24,27 & 11,05 & & 35,90 & 6,16 & \\
\hline Buenas & & & & & & & & & \\
\hline
\end{tabular}

* Prueba t de Student. 
Tabla 5. Proporción de SBO según variables estudiadas en alumnos del séptimo año de la facultad de medicina Alberto Hurtado - Universidad Peruana Cayetano Heredia, 2010.

\begin{tabular}{|c|c|c|c|c|}
\hline & \multirow[b]{2}{*}{ Variables } & \multicolumn{2}{|c|}{ Síndrome Burnout } & \multirow[b]{2}{*}{$\mathrm{p}^{*}$} \\
\hline & & Presente n, (\%) & Ausente n, (\%) & \\
\hline \multirow{2}{*}{ Sexo } & Masculino & $32(58,1)$ & $23(41,8)$ & \multirow{2}{*}{0,843} \\
\hline & Femenino & $27(56,2)$ & $21(43,7)$ & \\
\hline \multirow{2}{*}{ Relación Sentimental } & Sí & $30(57,6)$ & $22(42,3)$ & \multirow{2}{*}{0,932} \\
\hline & No & $29(56,8)$ & $22(43,1)$ & \\
\hline \multirow{2}{*}{ Convivencia } & Padres y/o otros familiares & $54(55,6)$ & $43(44,3)$ & \multirow{2}{*}{$0,235^{\ddagger}$} \\
\hline & Solo & $5(83,3)$ & $1(16,6)$ & \\
\hline \multirow{2}{*}{$\begin{array}{l}\text { Actividad } \\
\text { Extracurricular }\end{array}$} & Sí & $10(45,4)$ & $12(44,5)$ & \multirow{2}{*}{0,206} \\
\hline & No & $49(60,4)$ & $32(39,5)$ & \\
\hline \multirow{2}{*}{ Consumo de Alcohol } & Nunca a 1 vez a la semana. & $36(51,4)$ & $34(48,5)$ & \multirow{2}{*}{0,080} \\
\hline & Más de 1 vez a la semana. & $23(69,6)$ & $10(30,3)$ & \\
\hline \multirow{2}{*}{ Consumo de Tabaco } & Sí & $19(59,3)$ & $13(40,6)$ & \multirow{2}{*}{0,773} \\
\hline & No & $40(56,3)$ & $31(43,6)$ & \\
\hline \multirow{2}{*}{ Remuneración } & Sí & $51(56,6)$ & $40(43,3)$ & \multirow{2}{*}{0,484} \\
\hline & No & $8(66,6)$ & $4(33,3)$ & \\
\hline \multirow{2}{*}{ Horas de Trabajo } & Más de 8 Horas & $52(58,4)$ & $37(41,5)$ & \multirow{2}{*}{0,554} \\
\hline & Menos de 8 horas & $7(50,0)$ & $7(50,0)$ & \\
\hline \multirow{2}{*}{ Curso desaprobado } & Sí & $9(81,8)$ & $2(18,2)$ & \multirow{2}{*}{$0,111^{\ddagger}$} \\
\hline & No & $50(54,3)$ & $42(45,6)$ & \\
\hline \multirow{2}{*}{$\begin{array}{l}\text { Horas de estudio } \\
\text { semanales }\end{array}$} & Más de 10 hrs. & $29(50,0)$ & $29(50,0)$ & \multirow{2}{*}{0,090} \\
\hline & Menos de 10 hrs. & $30(66,6)$ & $15(33,3)$ & \\
\hline \multirow{2}{*}{ Horas de sueño diario } & Menos de 6 & $32(59,2)$ & $22(40,7)$ & \multirow{2}{*}{0,670} \\
\hline & Más de 6 & $27(55,1)$ & $22(44,8)$ & \\
\hline \multirow{4}{*}{$\begin{array}{l}\text { Presencia de } \\
\text { Enfermedad Mental } \\
\text { Relaciones con el } \\
\text { Equipo }\end{array}$} & Sí & $7(87,5)$ & $1(12,5)$ & \multirow{2}{*}{$0,134^{\ddagger}$} \\
\hline & No & $52(54,7)$ & $43(45,2)$ & \\
\hline & Muy buenas y buenas. & $54(55,6)$ & $43(44,3)$ & \multirow{2}{*}{$0,235^{\ddagger}$} \\
\hline & Malas y Regulares & $5(83,3)$ & $1(16,3)$ & \\
\hline
\end{tabular}

respuesta fue alta, pueden existir sesgos. Se desconoce en qué medida el estrés profesional y personal puedan afectar las respuestas, los estudiantes afectados pueden encontrarse menos motivados a llenar correctamente una encuesta; por otro lado, los mismos pueden tener mayor interés en participar ya que el tópico en cuestión es de relevancia para ellos.

Otro sesgo a tomar en cuenta es la mayor o menor sinceridad de los estudiantes para contestar los cuestionarios, pues estos se pudieron alterar por circunstancias tales como sobredimensionar las condiciones laborales o aparentar ser internos ideales. Además, la dependencia exclusiva en una escala autoadministrada puede contribuir al error de medida relacionado a las tendencias de respuesta positiva o negativa sistemáticas. Además consideramos que los estudiantes pudieron recordar con mayor precisión las experiencias vividas durante el periodo de tiempo más próximo al momento en que llenaron el cuestionario, de tal manera que se podría subestimar o sobreestimar su valoración en el mismo dependiendo de la rotación en la cual se encontraban al momento de finalizar su internado. Por último, el incluir tan solo un año de estudios y una universidad puede afectar los resultados obtenidos.

La fortaleza de nuestro estudio radica en haber utilizado un instrumento con propiedades psicométricas validadas para medir SBO, permitiendo una comparación con otras poblaciones de estudiantes, residentes y médicos. Nuestro estudio es el primero realizado en el país al respecto y puede ser la base para estudios más complejos. 
En conclusión, la elevada presencia de SBO en los internos de medicina de nuestro trabajo supone una alerta y plantea la necesidad de realizar estudios futuros al respecto que permitan dar a conocer los factores que ayudan a los estudiantes a recuperase del SBO. Conjuntamente las facultades de medicina deberían implementar un sistema para identificar a los estudiantes que podrían sufrir de SBO, optimizar el ambiente de estudio, mejorar la organización de las rotaciones clínicas, desarrollar programas de soporte, consejería estudiantil y brindar mayores servicios de salud mental para crear una cultura de bienestar que provea a los estudiantes de herramientas para promover su calidad de vida.

\section{Correspondencia:}

Carlos Renato Jiménez-Román

Dirección:

Av. Manuel Villarán 1045.

Los Sauces, Surquillo.

Lima 034. Perú.

Correo electrónico: quentasilmarill@gmail.com

Teléfonos: (511) 2731479 / 990185197.

\section{REFERENCIAS BIBLIOGRÁFICAS}

1. Dyrbye L, Thomas M, Shanafelt T. Medical student distress: Causes, consequences, and proposed solutions. Mayo Clin Proc 2005; 80: 1613-1622.

2. Pereyra R, Ocampo J, Silva V, Vélez E, Da Costa D, Toro $\mathrm{L}$, et al. Prevalencia y factores asociados con síntomas depresivos en estudiantes de ciencias de la salud de una universidad privada de Lima, Perú 2010. Rev Peru Med Exp Salud Pública 2010; 27: 520-26.

3. Galli E, Feijóo L, Roig I, Romero S. Aplicación del "MINI" como orientación diagnóstica psiquiátrica en estudiantes de medicina de la Universidad Peruana Cayetano Heredia: informe preliminar epidemiológico. Rev Med Hered 2002; 13:19-25.

4. Saavedra J. Estudio Epidemiológico Metropolitano en Salud Mental 2002. Informe general. Anales de Salud Mental 2002; 18 (Supl 1 y 2).

5. Vasquez F, Muñoz P. Prevalence of psychopathology in medical students at local, private university. En: Möller H, Rupprecht R, Hojaij C. (Editors). Proceedings of the Biology of Psychoses European Congress of Biological Psychiatry; 2002 Jun 13-16; Copenhagen Denmark. Scotland: East Kilbride, 2002.

6. Rodas P, Santa Cruz G, Vargas H. Frecuencia de trastornos mentales en alumnos del tercer año de la Facultad de Medicina de una universidad privada de Lima, 2006. Rev Med Hered 2009;20:70-76.
7. Dyrbye L, Thomas M, Massie S, Power D, Eacker A, Harper W, et al. Burnout and suicidal ideation among U.S. medical students. Ann Intern Med 2008; 149: 334341.

8. Thomas N. Resident burnout. JAMA 2004;292:28802889.

9. Albaladejo R, Villanueva R, Ortega P, Astacio P, Calle ME, Dominguez V. Sindrome de burnout en el personal de enfermería en un hospital de Madrid. Rev Esp Salud Publica 2004; 78: 505-516.

10. Álvarez-Hernández G, Medécigo-Vite S, Ibarra-García C. Prevalence of burnout syndrome in medical residents from a pediatric hospital in Sonora, Mexico - 2008. Bol Med Hosp Infant Mex 2010; 67:40-46.

11. Merino E. Síndrome de burnout en trabajadores del Servicio de Pediatría del Hospital Sullana, 2007. Lima: Universidad Peruana Cayetano Heredia; 2009.

12. Gamonal Y, García C, Silva Z. Síndrome de burnout en el profesional de enfermería que labora en áreas críticas. Rev Enferm Herediana 2008; 1: 33-39.

13. Maslach C, Jackson SE, Leiter MP. Maslach Burnout Inventory Manual. 3th ed. Palo Alto, CA: Consulting Psychologists Press; 1996.

14. Maslach C, Shaufeli W, Leiter M. Job burnout. Annu Rev Psychol 2001; 52:397-422.

15. Dyrbye L, West C, Shanafelt T. Defining burnout as a dichotomous variable. J Gen Intern Med 2009; 24:440.

16. Guido Evans F. Síndrome de burnout en residentes de cirugía y cirujanos generales de tres hospitales de Lima Metropolitana, 2006: un estudio piloto. Lima:

Universidad Peruana Cayetano Heredia; 2006.

17. Jamanca R, Vega C, Zanabria D. Sindrome de burnout en personal médico del Hospital Nacional Cayetano Heredia. Lima: Universidad Peruana Cayetano Heredia; 2007.

18. Guevara C, Henao D, Herrera J. Síndrome de desgaste profesional en médicos internos y residentes. Hospital Universitario del Valle, Cali, 2002. Colomb Med 2004; 35: 173-178.

19. Aguilar A, Gutiérrez R. El fenómeno de burnout en médicos en dos hospitales de Lima-Perú. Rev Psicol Herediana 2007; 2:1-13.

20. Dyrbye L, Thomas M, Harper W, Massie S, Power D, Eacker A, et al. The learning environment and medical student burnout: a multicentre study. Med Educ 2009; 43: 274-282.

21. Dyrbye L, Thomas M, Huschka M, Lawson K, Novotny P, Sloan J, et al. A Multicenter study of burnout, depression, and quality of life in minority and nonminority US medical students. Mayo Clin Proc 2006; 81: 1435-1442.

22. Santen S, Holt D, Kemp J, Hemphill R. Burnout in medical students: Examining the prevalence and associated factors. South Med J 2010; 103: 758-764. 
23. Dyrbye L, Power D, Massie S, Eacker A, Harper W, Thomas M, et al. Factors associated with resilience to and recovery from burnout: a prospective, multiinstitutional study of US medical students. Med Educ 2010; 44:1016-1026.

24. Willcock S, Daly M, Tennant C, Allard B. Burnout and psychiatric morbidity in new medical graduates. MJA 2004; 181: 357-360.

25. Borda M, Navarro E, Aun E, Berdejo H, Racedo K, Ruiz J. Síndrome de burnout en estudiantes de internado del Hospital Universidad del Norte. Salud Uninorte 2007; 23:43-51.

26. García J, Herrero S, León J. Validez factorial del MBI en una muestra de trabajadores del Hospital Psiquiátrico Penitenciario de Sevilla. Apuntes de Psicología 2007; 25: 157-174.

27. Manso-Pinto J. Estructura factorial del Maslach Burnout Inventory - Version Human Services Survey en Chile. Rev Interamer Psicolog 2001; 40: 115-118.

28. Olivares V. Análisis de las propiedades psicométricas del Maslach Burnout Inventory Human Services (MBI-
HSS) en profesionales chilenos. Cienc Trab 2009; 11: 217-221.

29. Gil-Monte P. Factorial validity of the Maslach Burnout Inventory (MBI-HSS) among Spanish professionals. Rev Saúde Pública 2005; 39: 1-8.

30. Schaufeli W, Bakker A, Hoogdwin K, Schaap C, Kladle A. On the clinical validity of the Maslach Burnout inventory and the burnout measure. Psychol Health 2001; 16: 565-82.

31. Jennings M. Medical student burnout: Interdisciplinary exploration and analysis. J Med Humanit 2009; 30: 253269.

32. Dyrbye L, Thomas M, Power D, Durning S, Moutier C, Massie S, et al. Burnout and serious thoughts of dropping out of medical school: A multi-institutional study. Acad Med 2010; 85:94-102.

33. Dahlin M, Runeson B. Burnout and psychiatric morbidity among medical students entering clinical training: A three year prospective questionnaire and interview-based study. BMC Med Educ 2007; 7: 6-14. 